\title{
Different tolerance of zooplankton communities to insecticide application depending on the species composition.
}

\author{
Masaki Sakamoto $^{1 *}$ and Yoshinari Tanaka ${ }^{2}$ \\ ${ }^{1}$ Faculty of Engineering, Toyama Prefectural University, 5180 Kurokawa Imizu-shi, Toyama 939-0398, Japan \\ ${ }^{2}$ Center for Environmental Risk Research, National Institute for Environmental Studies, Onogawa 16-2, Tsukuba 305-8506, Japan
}

\begin{abstract}
Natural zooplankton communities are composed of many different species at different trophic levels in the aquatic food web. Several researchers have reported that in mesocosm/enclosure experiments, larger cladocerans tend to be more sensitive to carbamate insecticides than smaller ones (Daphnia > Moina, Diaphanosoma > Bosmina). In contrast, results from individual-level laboratory tests have suggested that large cladoceran species are more tolerant than small species. To clarify this inconsistency, we conducted a microcosm experiment using model zooplankton communities with different species compositions, where animals were exposed to lethal (near to the $24 \mathrm{~h}$ LC50, concentration estimated to kill $50 \%$ of individuals within 24-h for the small cladoceran Bosmina) and lower, sublethal concentrations of carbaryl. In the experiment, population densities of the small cladocerans (Bosmina and Bosminopsis) decreased subsequent to the applications of chemical, but no impacts were observed on the large cladoceran Daphnia. Our results supported the reports of previous individual level toxicity tests, and indicated that the sensitivity of zooplankton to the insecticide was unchanged by biological interactions but the response of population can be modified by compensation of population through hatching from resting eggs and/or the persistence of insecticide in the systems.
\end{abstract}

Keywords: insecticide, microcosm, zooplankton

\section{INTRODUCTION}

Anthropogenic toxic chemicals can easily contaminate water bodies, and exert negative impacts on the ecosystems. Reference indexes for standard test organisms, such as NOEC (No observed effect concentration) and EC50 (50\% effective concentration, meaning the concentration estimated to immobilize $50 \%$ of individuals) values obtained from laboratory toxicity tests (OECD 2004), have been used for the estimation of direct toxic impacts on target trophic level biocenose. On the other hand, community level experiments have elucidated indirect impacts of the chemicals through the modification of biological interactions (Preston 2002, Van Wijngaarden et al. 2005,
Relyea and Hoverman 2006, Sarma and Nandini 2006).

Lake ecosystems are composed of many different species at different trophic levels. Cladocerans (zooplankton) are one of the groups most sensitive to insecticides. They are, however, superior herbivores in food competition with rotifers (MacIsaac and Gilbert 1989). Community level mesocosm/enclosure experiments have demonstrated that rotifer density increases after disruption of cladoceran populations at insecticide-contaminated sites (Chang et al. 2005). This is because rotifers are far more tolerant than cladocerans to insecticides (Havens and Hanazato 1993). Within the cladoceran group, results

\section{Open Access http://dx.doi.org/10.5141/ecoenv.2013.018}

This is an Open Access article distributed under the terms of the Creative Commons Attribution Non-Commercial License (http://creativecommons. org/licenses/by-nc/3.0/) which permits unrestricted non-commercial use, distribution, and reproduction in any medium, provided the original work is properly cited.
Received 15 April 2013, Accepted 18 June 2013

*Corresponding Author

E-mail: masaki@pu-toyama.ac.jp

Tel: +81-766-56-7500 
from mesocosm/enclosure experiments have suggested that large cladocerans tend to be more sensitive to insecticides than smaller ones (e.g., Daphnia > Moina, Diaphanosoma > Bosmina) (Hanazato 1991a, Havens 1994, Hanazato and Kasai 1995). This implies that zooplankton communities dominated by large cladocerans are more vulnerable to the toxins than those dominated by small ones. In natural environments, the size structure of zooplankton is governed by bottom-up and top-down forces (Finlay et al. 2007). Small cladocerans such as Bosmina often dominate the zooplankton communities in eutrophic and fish-abundant lakes. On the other hand, large Daphnia commonly become abundant in oligotrophic lakes, where fish biomass is not very high. Therefore, it might be considered that oligotrophic plankton communities are more vulnerable to the contamination by those insecticides.

In contrast to the community level experiments, however, results from the individual-level laboratory tests have suggested that Bosmina are more vulnerable to insecticide than Daphnia (Passino and Novak 1984, Sakamoto et al. 2005). Within genus Daphnia, similarly, smallbodied species tends to be more sensitive to the chemicals (Pereira and Gonçalves 2007 Vesela and Vijverberg 2007). The negative correlation between body size and the sensitivity to the toxic chemicals seem to be stronger in particular families (Bossuyt and Janssen 2005).

The contradiction between community and individual level studies indicates that the vulnerability of each cladoceran species to insecticide can change greatly when a variety of species coexist and interact in a complex manner. However, there is insufficient information to explain the phenomenon.

Insecticides often affect the plankton communities indirectly by the modification of predator-prey interactions (Lürling and Scheffer 2007). For instance, increased swimming speed of the rotifer, Brachionus, induced by pentachlorophenol, increases its encounter rate with invertebrate predators, resulting in its increased vulnerability to predation (Preston et al. 1999). Induction of morphological defense of some Daphnia species, normally mediated by natural organic chemicals, is enhanced on exposure to some insecticides (carbaryl, BPMC, temephos, diazinon) (Hanazato 1991b, 1995, Hanazato and Dodson 1993). Conversely, Sakamoto et al. (2006, 2009) have reported that carbaryl inhibits the development and maintenance of anti-predator morphology in Bosmina. Therefore, the manner of disturbance of the predatory interaction differs depending on the species/genus, even if the animals belong to the same biological group. The degree of influ- ence of such indirect effects of insecticides on the population dynamics of each species and on the community structure are unproved by the community level studies, because those phenomena have only been observed in individual- or population-level experiments.

A carbamate insecticide, carbaryl (1-naphthyl-Nmethylcarbamate), was used in the present study since its toxicity to cladocerans had been well tested both in species- and community level studies (Hanazato 2001). Here, we investigated the following two hypotheses by a microcosm experiment: (1) the response to carbaryl of each cladoceran changes in the presence of competitive or predatory interactions; (2) disturbance of the predatory interactions is a possible cause yielding the inconsistency between community- and individual-level studies.

In order to compare the tolerances of plankton communities with different structures, two different zooplankton communities, dominated by Bosmina or Daphnia, were prepared. These were exposed to sub lethal or lethal effective concentrations of carbaryl.

\section{MATERIALS AND METHODS}

Six $20 \mathrm{~L}$ cylindrical polyethylene tanks (diameter, 30 $\mathrm{cm}$; height, $31 \mathrm{~cm}$ ) were used as microcosms for the preparation of zooplankton communities 14 days before the treatments (day -14). The zooplankton community was established from the resting eggs (or the resting stages of the animals) in bottom mud obtained from the central area of a eutrophic lake, Lake Suwa $\left(36^{\circ} 2^{\prime} \mathrm{N}, 138^{\circ} 5^{\prime} \mathrm{E}\right) \mathrm{Ja}-$ pan. In Lake Suwa, the zooplankton community is dominated by bosminid species, and no Daphnia are observed. Approximately $500 \mathrm{~g}$ dry weight of mud was placed in each microcosm tank with $20 \mathrm{~L}$ dechlorinated tap water. The tanks were kept under constant conditions $\left(21 \pm 1^{\circ} \mathrm{C}\right.$; $14 \mathrm{~h}$ light, $10 \mathrm{~h}$ dark). The green alga, Chlorella vulgaris (Chlorella Industry Co. Ltd., Fukuoka, Japan, $0.58 \mu \mathrm{g}$ C m $\left.\mathrm{L}^{-1}\right)$ was introduced to the tanks as food for the zooplankters on day $-10,-6$ and every third day thereafter. On day 0 , all of the water in the six tanks was transferred to a 500 L polycarbonate container by siphoning with a silicon rubber tube. After gentle stirring, seven liter of the pooled water containing zooplankters was poured into each of 15 polyethylene tanks, and then diluted by adding $13 \mathrm{~L}$ of dechlorinated tap water (total volume, $20 \mathrm{~L}$ ). Therefore, the microcosm tanks contained equalized model zooplankton community and no bottom mud (egg bank). To create two different plankton communities, twelve adult females of laboratory-cultured Daphnia galeata (single 


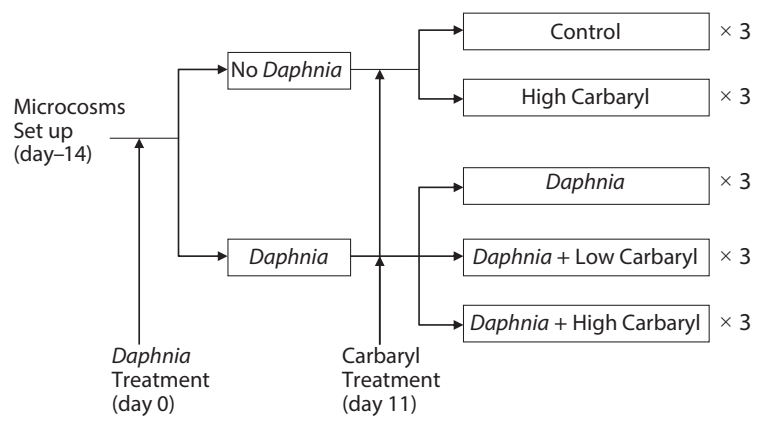

Fig. 1. Experimental procedure: Daphnia and carbaryl applications were made on day 0 and day 11 , respectively.

clone, originally from Lake Kasumigaura Japan) were added into each of the 9 tanks (Fig. 1).

Carbaryl applications were conducted on day 11 . The insecticide ( $>99 \%$ grade, CAS $63-25-2)$ was purchased from Wako Pure Chemical Industries Ltd., Japan. A stock solution of the reagent $(1000 \mathrm{mg} / \mathrm{L})$ was prepared by dissolving the chemical in $99 \%$ ethanol to a final volume of $10 \mathrm{~mL}$, then diluted to the required concentrations with dechlorinated tap water, and introduced to the tanks. The established zooplankton communities were exposed to following five different treatments, each having three replications: (1) control (no carbaryl and no Daphnia); (2) high carbaryl treatment ( $5 \mu \mathrm{g} / \mathrm{L}$ of carbaryl, nominal concentration), (3) Daphnia treatment; (4) Daphnia and low carbaryl treatment ( $2 \mu \mathrm{g} / \mathrm{L})$; (5) Daphnia and high carbaryl treatment $(5 \mu \mathrm{g} / \mathrm{L})$ (Fig. 1). For the treatments (1), (3) and (4), Ethanol (the solvent used for carbaryl) was added to equalize the solvent concentration $(5 \mu \mathrm{L} / \mathrm{L})$ in the treatments. The maximum carbaryl concentration (5 $\mu \mathrm{g} / \mathrm{L}$ ) was nearly equal to the $24 \mathrm{~h} \mathrm{LC50}$ values (B. fatalis, $4.1 \mu \mathrm{g} / \mathrm{L} ;$ B. longirostris, $8.6 \mu \mathrm{g} / \mathrm{L}$ ) for Bosmina, the dominant cladoceran in Lake Suwa (Sakamoto et al. 2005), but lower than the $48 \mathrm{~h}$ LC50 value $(11.3 \mu \mathrm{g} / \mathrm{L})$ for the $D$. $g a$ leata clone used in this study (Mano et al. 2010).

To measure the absolute concentrations of carbaryl, $100 \mathrm{~mL}$ water was collected from each tank at 0, 3, 6 and 9 days after the application (day 11 to 20). Each water sample containing carbaryl was filtered through a Whatman GF/C filter, and passed through a solid-phase cartridge (PS-2 plus; Waters, Milford, MA, USA). The samples were eluted with $5 \mathrm{~mL}$ acetonitrile. The acetonitrile solutions were dried under a gentle nitrogen stream and redissolved in $500 \mu \mathrm{L}$ acetonitrile for HPLC analysis. Parent carbaryl concentrations were determined by HPLC (LC-10A series; Shimadzu, Kyoto, Japan) with UV-VIS detector (SPD-10A; Shimadzu, Kyoto, Japan) equipped with an ODS column (Mightysil RP-18 GP $150 \mathrm{~mm} \times 2.0 \mathrm{~mm} \phi(5 \mu \mathrm{m})$; Kanto
Chemical Co., Inc., Tokyo, Japan).

Zooplankters were sampled before carbaryl application on days 0 and 11, and every third day thereafter by using a column sampler (diameter $5.5 \mathrm{~cm}$, length $50 \mathrm{~cm}$ ) with a hydraulically operated flap at the bottom (total volume: $1 \mathrm{~L}$ ). Just before the samplings, $\mathrm{DO}$ and $\mathrm{pH}$ were monitored for each microcosm tanks. Collected water was filtered through a $40-\mu \mathrm{m}$ mesh and the residue was fixed with sugar-containing formalin at a final concentration of $4 \%$ (Haney and Hall 1973). The fixed samples were concentrated to $5 \mathrm{~mL}$ by settling for over $6 \mathrm{~h}$. Aliquots of $1 \mathrm{~mL}$ were used for counting rotifers and copepod nauplii. Whole samples were used for counting cladocerans and copepods. Zooplankton were identified to species (or genus) level and counted using a microscope. In addition, morphotypes (antennule types) of adult females of B. longirostris were identified following the criteria used previously by Sakamoto et al. (2007), where animals with hooked antennules were identified as 'cornuta' and ones with slightly curved antennules as 'pellucida (defensive morph)'.

Effects of the treatments on the repeatedly sampled zooplankton abundances, the morphologies of Bosmina, physico-chemical data (dissolved oxygen (DO) and $\mathrm{pH}$ ) were tested with repeated-measures ANOVA using StatView ver. 5 (SAS Institute Inc., Cary, NC, USA). The morphotype data was log-transformed before the tests.

For each microcosm, a time-weighted average (WA) was calculated for each response variable (carbaryl concentration, densities of zooplankton, relative abundance of "pellucida"-morphed B. longirostris) using the following formula (Stephen et al. 2004):

$$
\begin{gathered}
\mathrm{WA}=\left[\left(11 \times V_{11}\right)+\left(14 \times V_{14}\right)+\left(17 \times V_{17}\right)+\right. \\
\left.\left(20 \times V_{20}\right)\right] /(11+14+17+20),
\end{gathered}
$$

Where $V$ is the response variable at each of the four sampling dates (day 11 to 20) weighted by sequential numbers to give increasing emphasis on each subsequent date and so account for temporal effects.

Model selections using a generalized linear model (GLM) with WA variables were performed to assess the effects of carbaryl and Daphnia on response variables $\left(\mathrm{WA}_{\mathrm{RES}}\right)$, using:

$$
\mathrm{WA}_{\mathrm{RES}}=X_{C A} C_{C A}+X_{D A} C_{D A}+b,
$$

Where $C_{C A}$ and $C_{D A}$ are WA values of carbaryl concentration and Daphnia density, respectively, and $X$ and $b$ indicate their parameter coefficients and the constant, respectively. Daphnia density was treated as an independent variable because Daphnia is a superior competitor 

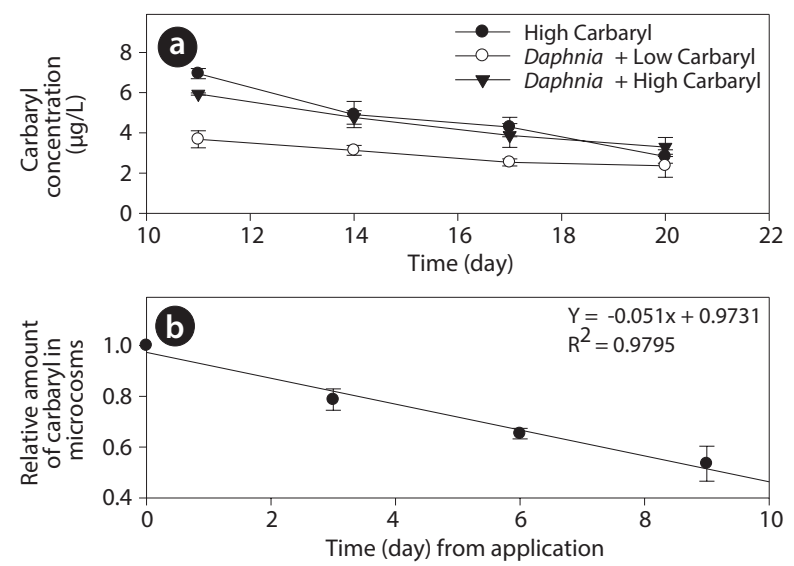

Fig. 2. Carbaryl concentrations (average $\pm S E$ ) in microcosms: (a) Measured concentrations by using HPLC. Decomposition rate of carbaryl (b) was approximately $5 \%$ per day, irrespective of either the presence of Daphnia in the community or the initial concentration of the insecticide.

to the other zooplankton (MacIsaac and Gilbert 1989).

To analyze the differences in species compositions and those abundances between no-carbaryl tanks and carbaryl-introduced tanks, principal response curves (PRCs) were calculated based on a redundancy analysis (RDA) (Van den Brink and Ter Braak 1999, Hens et al. 2005) by using “Vegan” package in R 2.15.2 (R Core Development Team 2011) . Biotic data (abundance) were $\log (+1)$ transformed, centered over time and standardized before the analysis. The environmental variable was each treatment, with sampling time as the co-variable. PRCs were derived by plotting the canonical coefficients $\left(\mathrm{C}_{\mathrm{dt}}\right)$ against time. The line at $y=0$ represents the mean of the controls and the $\mathrm{C}_{\mathrm{dt}}$ 's of the treated microcosms indicate their deviation from the controls for each sampling date. Accompanying species scores allow an interpretation nat the species level. Data sets for no-Daphnia treatments and Daphnia-introduced treatments were analyzed separately in order to avoid interference of Daphnia data in the $\mathrm{C}_{\mathrm{dt}}$ 's of No-Daphnia treatments. Quantitative difference between the treatments was tested with a Monte Carlo permutation test.

All statistical analyses excepting repeated-measures ANOVA were performed using the statistical software $R$ (version 2.15.2).

\section{RESULTS}

During the experiment, $\mathrm{DO}$ and $\mathrm{pH}$ in tanks ranged between 3-7 $\mathrm{mg} \mathrm{O}_{2} \mathrm{~L}^{-1}$ and 6.7 to 7.2, respectively. However, the values did not differ significantly among the treat- ments $(P>0.05)$. Carbaryl concentrations in the treatment tanks were slightly higher than the nominal values (Fig. 2a). The initial concentrations (average \pm SE) were $6.95 \pm 0.27,3.68 \pm 0.43$ and $5.93 \pm 0.06 \mu \mathrm{g} / \mathrm{L}$ in the "high carbaryl", "Daphnia + low carbaryl" and "Daphnia + high carbaryl" treatments, respectively. The absolute reduction of the concentration was $5 \%$ per day irrespective of either the presence of Daphnia or the initial concentration of the insecticide (Fig. 2b).

Carbaryl application caused a downdrift of the cladoceran density dependent on the concentrations (Fig. 3a). Contrary to the cladocerans, total herbivorous rotifer density increased markedly in the "high carbaryl" treatment (Fig. 3b). However, densities of the carnivorous zooplankton did not differ between the treatments (Fig. 3c and $3 \mathrm{~d})$.

Cladoceran communities were mainly composed of two Bosmina species (B. fatalis and B. longirostris) and Bosminopsis deitersi (excepting the introduced D. galeata) (Fig. 4a-4c). Depending on the concentrations of carbaryl applied, their populations were subsequently disrupted $(P<0.01$ for treatment, time and treatment by time interaction). However, $B$. longirostris was not affected severely by the low carbaryl treatment. A negative impact of carbaryl on the densities of $D$. galeata was not observed, even in the high dose treatment (Fig. 4d). Filinia longiseta and Hexarthra mira were the most abundant herbivorous rotifers in the microcosms (Fig. 4e and 4f). Total density of the rotifers tended to increase when they were exposed to the high carbaryl concentration treatments, except in the Daphnia-introduced microcosms (Fig 4e and 4f). Lepadella was the only rotifer that formed large populations in the presence of Daphnia (Fig. 4g). Copepod nauplii were not affected by the carbaryl applications (Fig. 4h). The relative abundance of $B$. longirostris having the defensive morphotype decreased when they were exposed to carbaryl (Fig. 4i).

We performed the model selections using a generalized linear model (GLM) with time-weighted average (WA) of response variables to assess the effects of carbaryl and Daphnia (Table 1 and Fig. 5). Null models (intercept only) were selected for D. galeata, Calanoida and Cyclopoida, i.e., they were not affected by the treatments (Table 1). Total cladocerans excepting Daphnia, B. fatalis, B. longirostris and Bosminopsis deitersi were negatively correlated with the carbaryl concentration but not with Daphnia. For the copepod nauplii and Asplanchna sieboldi, a negative correlation was detected only with Daphnia density. However, the correlation for nauplii was not significant ( $P>0.05$ with t-test). Full models (interaction with both 


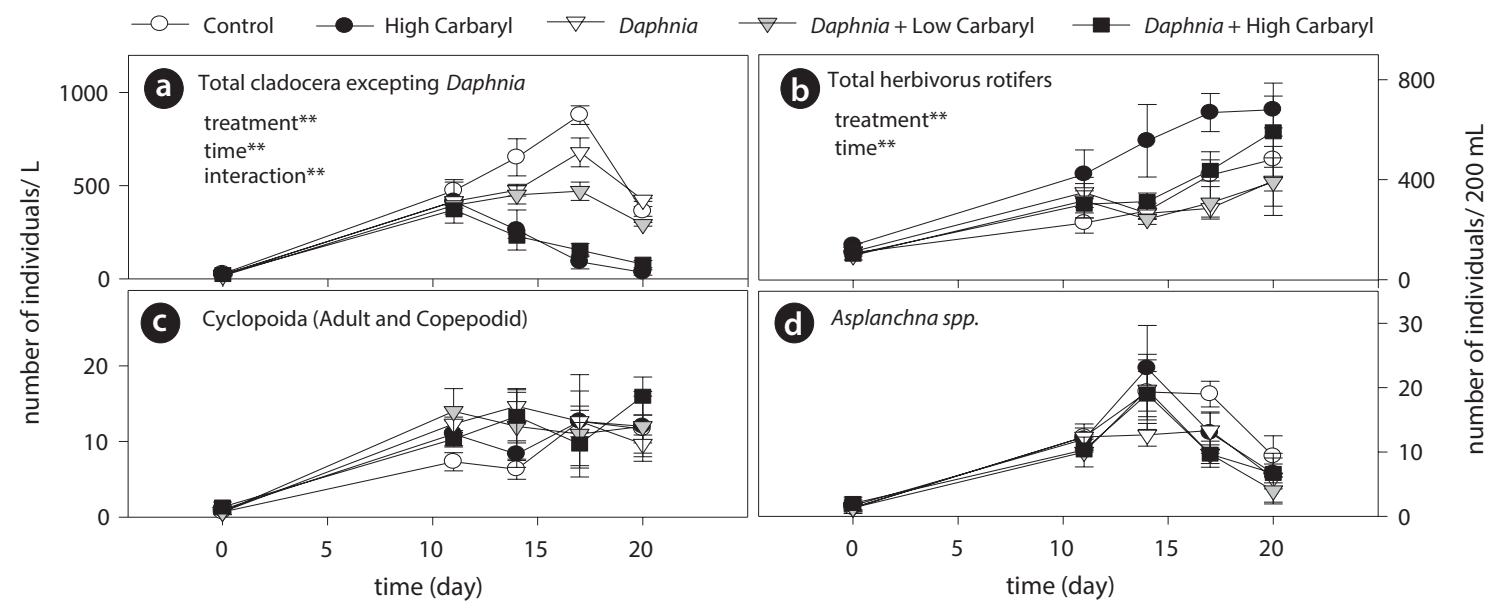

Fig. 3. Changes in density of herbivorous (a and b) and carnivorous ( $c$ and d) zooplankton in each treatment (average \pm SE). Asterisks $(*)$ show the effects of explanatory variables $(*, P<0.05 ; * *, P<0.01$ with repeated-measures ANOVA. No asterisk means $P \geq 0.05)$.

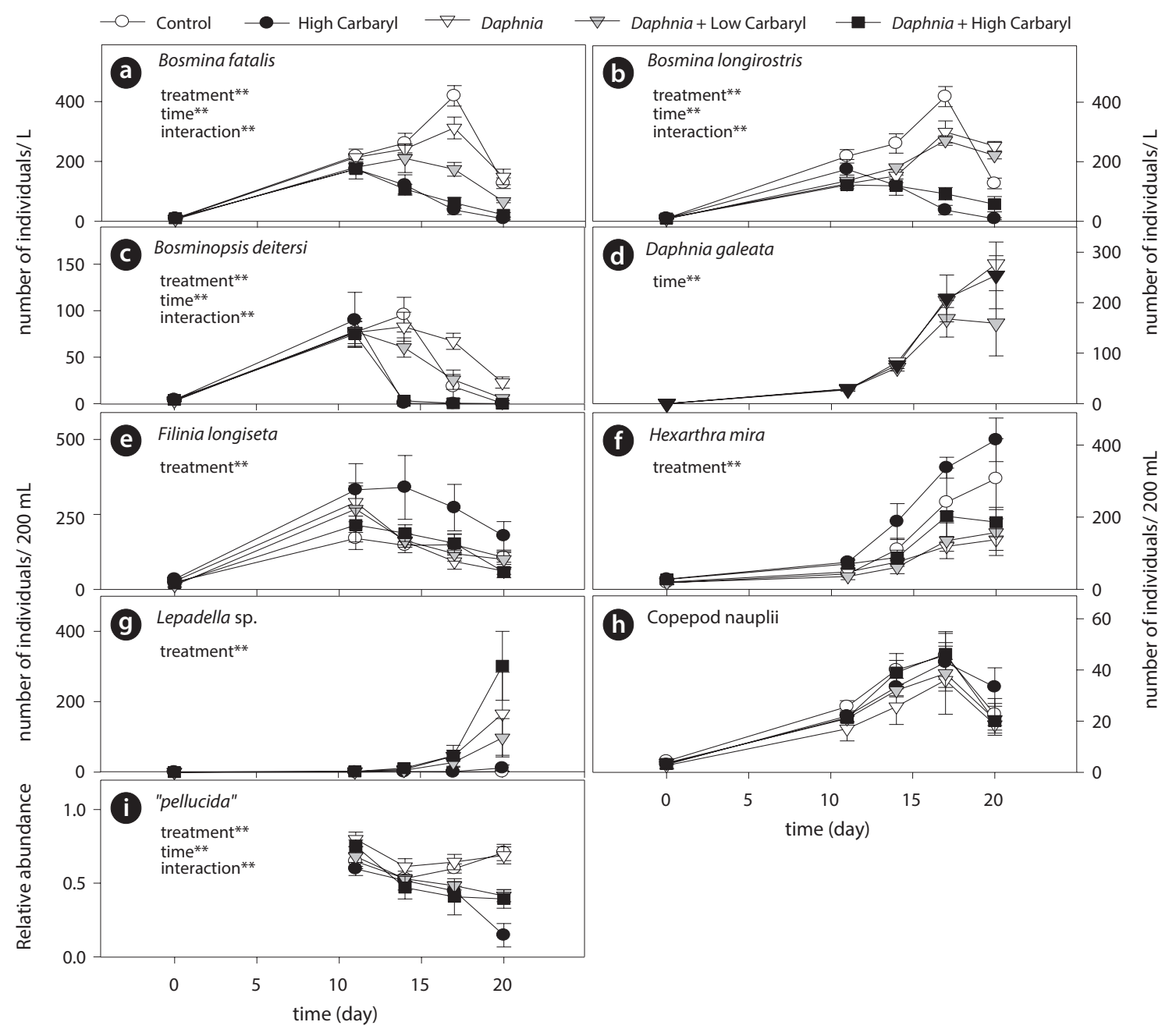

Fig. 4. Changes in density (average $\pm \mathrm{SE}$ ) of herbivorous cladocerans (a-d) and rotifers (e-g), copepod nauplii (h) and relative abundance of "pellucida (defensive)"-morphed B. longirostris in adult females (i) in each treatment. Note that $Y$-axes rotifer and nauplii (e-h) are represented as individual numbers per $200 \mathrm{~mL}$. Asterisks $\left(^{*}\right)$ show the effects of explanatory variables $\left({ }^{*}, P<0.05 ;{ }^{* *}, P<0.01\right.$ with repeated-measures ANOVA. No asterisk means $\left.P \geq 0.05\right)$. 
carbaryl and Daphnia) were selected for the total herbivorous rotifers, $H$. mira and F. longiseta. However, the negative impact of Daphnia on the total rotifer density

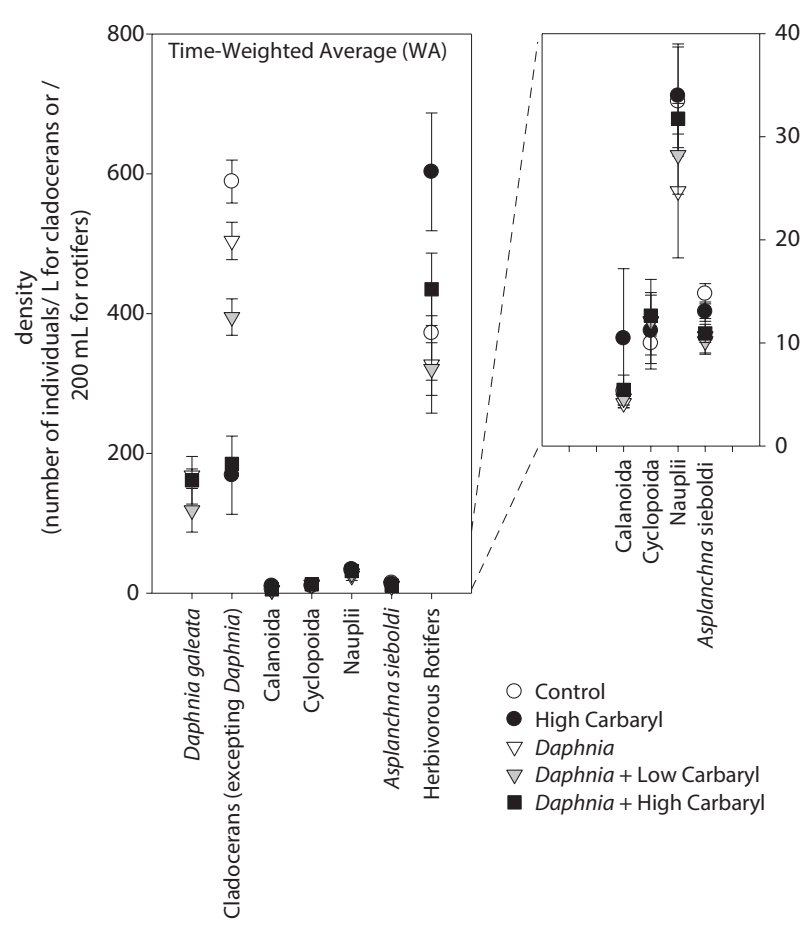

Fig. 5. Time-weighted averages (WA) of zooplankton in each treatment (average density $\pm \mathrm{SE}$ ). was not significant. The positive effect of carbaryl on rotifers was not significant for species level values. Daphnia density was positively correlated only with Lepadella density. The relative abundance of "pellucida"-morphed B. longirostris was negatively correlated with the carbaryl concentration.

PRC provided the information about the changes in species (taxon) composition due to the carbaryl applications (Fig. 6). The first components of PRC in no-Daphnia treatments and Daphnia-introduced treatments explained more than half of the variance $(71.3 \%$ and $69.5 \%$, respectively), so only one panel for each are shown. Effects of carbaryl applications were significant for both noDaphnia treatments and Daphnia-introduced treatments $(P<0.01$ with Monte Carlo test). Canonical coefficients $\left(\mathrm{C}_{\mathrm{dt}}\right)$ in day 11 were close to zero, indicating that the species composition did not differ between the treatments at that time. The large impact of carbaryl was detected during first 6 days (day 11 to 17) in the "high carbaryl" and "Daphnia + high carbaryl" treatments (Fig. 6). In "Daphnia + low carbaryl" treatment, $\mathrm{C}_{\mathrm{dt}}$ did not fluctuate until day 14 and slightly increased thereafter. Negative species scores occurred for the cladoceran species excepting Daphnia, and positive for the herbivorous rotifers in the no-Daphnia treatments (Fig. 6a). Negative scores indicate that zooplankton decreased due to the carbaryl exposure. A similar trend was observed in the Daphnia-introduced treatments (Fig. 6b).

Table 1. Generalized linear model (GLM) used to estimate the effects of carbaryl concentration and Daphnia density on the response variables.

\begin{tabular}{|c|c|c|c|c|c|}
\hline \multirow{2}{*}{ Variables } & \multicolumn{3}{|c|}{ Parameter coefficien in best model } & \multicolumn{2}{|c|}{ AIC } \\
\hline & Intercept & Carbaryl & Daphnia & Best & Full \\
\hline D. galeata & 89.69 & - & - & 178.36 & 180.36 \\
\hline Cladocerans $^{* 1}$ & 557.17 & -82.11 & - & 173.82 & 175.13 \\
\hline B. fatalis & 239.78 & -36.64 & - & 148.72 & 150.09 \\
\hline B. longirostris & 162.14 & 267.04 & -37.89 & - & 161.09 \\
\hline B. deitersi & 50.32 & -7.57 & - & 114.11 & 114.80 \\
\hline Calanoida & 6.00 & - & - & 94.64 & 96.30 \\
\hline Cyclopoida & 11.60 & - & - & 87.60 & 91.18 \\
\hline Nauplii & 33.49 & - & -0.03 & 106.98 & 108.33 \\
\hline Asplanchna & 13.58 & - & -0.02 & 67.34 & 68.18 \\
\hline Herb. Rotifers & 398.79 & 35.61 & -0.77 & 189.05 & - \\
\hline H. mira & 202.66 & 13.10 & -0.73 & 172.21 & - \\
\hline F. longiseta & 164.75 & 15.76 & -0.39 & 167.93 & - \\
\hline Lepadella sp. & 5.38 & - & 0.44 & 163.48 & 164.85 \\
\hline “pellucida"*2 & 0.57 & -0.13 & - & 91.63 & 92.14 \\
\hline
\end{tabular}

*1: total cladocerans excepting Daphnia; *2: the relative abundance of "pellucida"-typed (defensive morphotype) individuals in adult females of Bosmina longirostris.

Selection of the best model was based on Akaike information criterion (AIC). 'Full' and 'Best' indicate AIC values of the full and the best model, respectively. Significant factors in bold $(P<0.05$ with t-test). 

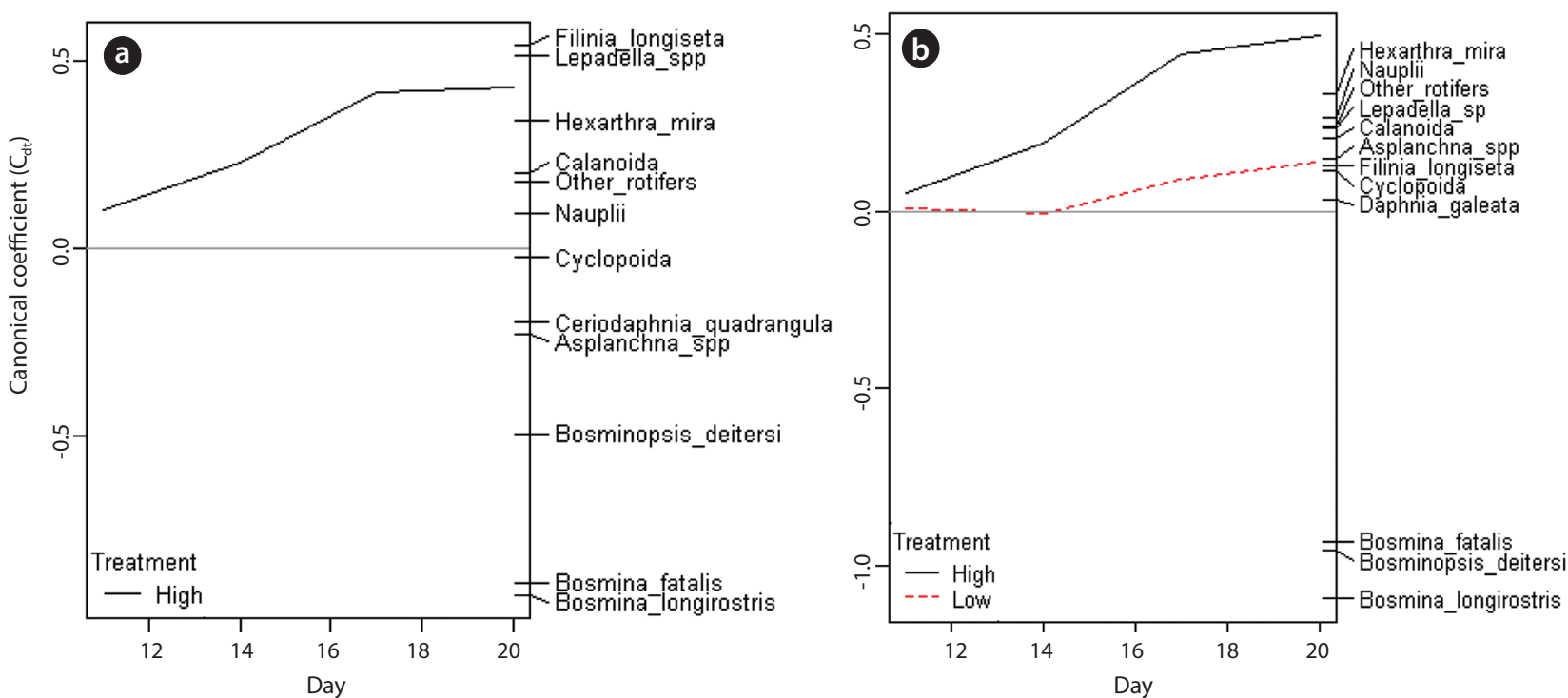

Fig. 6. Principal response curves (PRC) with species weights for the zooplankton data set, (a) no-Daphnia treatments, (b) Daphnia-introduced treatments, indicating the effects of low and high dose of the insecticide. The canonical coefficients ( $C_{\mathrm{dt}}$ 's, first principal component of treatment effects) of the treated microcosms indicate their deviation of species composition from the controls for each sampling date. $71.3 \%$ and $69.5 \%$ of variance were explained by the first components in the left and right panels, respectively.

\section{DISCUSSION}

Population dynamics of zooplankton in lakes and ponds are controlled by complex combinations of biotic interactions and abiotic environmental factors. Vulnerability to anthropogenic toxic chemicals differs between each species, so the chemicals should induce different community structures. Several researchers have concluded that large cladocerans tend to be more sensitive to insecticides than smaller ones based on mesocosm/enclosure experiments (Moore and Folt 1993, Hanazato 1998). On the other hand, the opposite relationship has been observed in the laboratory toxicity tests (Passino and Novak 1984, Sakamoto et al. 2005). However, the "size-dependent-tolerance" is not always applicable to broader taxonomic scale comparison (Mano et al. 2010).

Results of the present study did not coincide with the previous community-level studies. The population densities of the small cladocerans (Bosmina and Bosminopsis) showed concentration-depending decline patterns subsequent to the chemical applications, but no impacts were observed in the large Daphnia (Table 1, Fig. 4 and Fig. 5). This supports the reports from the individual level toxicity tests, and indicates that the vulnerability of each cladoceran to insecticide does not change greatly even when a variety of species coexist with complex interactions.
A possible reason for the above inconsistency is the rapid recovery of the small cladocerans by hatching from resting eggs in the enclosure studies. Hanazato (1991a) reported that Bosmina populations were affected by carbaryl, but recovered soon. The carbaryl concentration of $100 \mu \mathrm{g} / \mathrm{L}$ he used was far higher than the $24 \mathrm{~h}$ LC50 values for Bosmina, $8.6 \mu \mathrm{g} / \mathrm{L}$ for B. longirostris and $4.1 \mu \mathrm{g} / \mathrm{L}$ for B. fatalis (Sakamoto et al. 2005). In the present study, we intentionally eliminated the bottom mud, including the resting eggs of zooplankton, from the microcosms. Thus the population dynamics of zooplankton were governed simply by the biological interactions between the planktonic individuals and the contamination of carbaryl. Moreover, the period of our observations was very short (9 days from the chemical application). Those were the considerable differences to previous mesocosm/enclosure experiments.

The difference in the results may also have been due to the different locations of the experiments. Carbaryl seems to be photolysed easily (Pérez-Ruiz et al. 2003) and the light intensity would be higher in field than in the laboratory. Hanazato and Yasuno (1990) reported that $89 \%$ of carbaryl applied to the outdoor enclosures was decomposed within 1 day. In our experiment, however, the absolute reduction of the concentration was only $5 \%$ per day irrespective of the initial concentration (Fig. 2). Different decomposition rates of carbaryl can lead to different re- 
sults, because the damaged reproductivity of the animals can recover if the concentration of toxic chemicals decreases.

One conspicuous effect of insecticide application on zooplankton communities is an increase of rotifer density (Chang et al. 2005). This is because rotifers are far more tolerant to the insecticides than cladocerans. Cladocerans show superior exploitative competition to rotifers, and therefore chemical pollution can free the latter from food shortage (Hanazato 2001). The same phenomenon was also observed in the present microcosm experiment. Total rotifer density was positively correlated with the carbaryl concentration (Table 1). However, this was not the case in the presence of $D$. galeata (Fig. 4). D. galeata was not affected even in the high dose treatments, so that the rotifers did not get relief from competition. Moreover, large cladocerans (especially Daphnia) are known to kill rotifers by their feeding activity (interference competition: Gilbert 1988). However, one rotifer, Lepadella sp., increased only when Daphnia was abundant (Table 1, Fig. $4 \mathrm{~g}$ ), so the response of rotifers to Daphnia differs depending on the species.

Negative correlation with Daphnia density was also found for the abundance of a carnivorous rotifer, $A$. sieboldi (Table 1). However, there would be no predatory interaction between them because Daphnia has too large body to be handled by A. sieboldi. They probably responded to the decrease of herbivorous rotifers (main food items), indicating that Daphnia indirectly suppressed the abundance of A. sieboldi. Densities of calanoid- and cyclopoid copepods did not vary between the treatments (Table 1, Fig. 3c and 3d). Chang et al. (2005) confirmed that the feeding activity of the copepods was not affected by $500 \mu \mathrm{g} / \mathrm{L}$ carbaryl. It can be concluded that copepods feed effectively on a wide range of food items and accommodate to the changes in their food source.

Zooplankton community structure diverged depending on the pollutant level (Fig. 6). In the high-dose treatments (Fig. 6), the canonical coefficient $\left(\mathrm{C}_{\mathrm{dt}}\right)$ of PRCs increased subsequent to the carbaryl application. This indicates that the decrease of sensitive species due to the acute toxicity of carbaryl and the incidental increase of rotifers occurred rapidly. In the low dose treatment, on the other hand, $\mathrm{C}_{\mathrm{dt}}$ value did not fluctuate from zero until day 14 , meaning that the direct lethal effect was weak. Sakamoto et al. (2009) have reported that the population growth rate of $B$. longirostris declined to $87 \%$ when the animals were exposed to carbaryl at $2 \mu \mathrm{g} / \mathrm{L}$, but this concentration had no impact on the bosminid survival. The time lag observed in the low carbaryl treatment might be caused by the reduction of reproductive rates in Bosmina and Bosminopsis (chronic toxic impacts). Despite the dose-related responses that were recorded, species level vulnerability to carbaryl did not change depending on the chemical concentration.

Insecticides not only affect the individual survival or the reproduction rate, but also disturb the prey-predator interactions by enhancing or inhibiting the induction of morphological defences of prey animals (Lürling and Scheffer 2007). For example, carbamate and organophosphorus insecticides enhance the development of protuberant morphologies of Daphnia normally induced by predator kairomones (chemicals released from predators) (Hanazato and Dodson 1993). Inducible morphological defense is an important device of herbivorous zooplankton for reducing the risk of extinction in the presence of predators. Altered morphology of prey species works as practical hedge, especially against size-dependent predation, by interfering with the predators' handling and ingestion (Tollrian and Dodson 1999). In contrast to Daphnia, carbaryl inhibits the induction or maintenance of defensive morphs in Bosmina (Sakamoto et al. 2006, 2009). In the present study, the relative abundance of $B$. longirostris having the morphotype defensive against cyclopoid copepods declined after the carbaryl treatments (both high and low) (Fig. 4i). Population density of $B$. longirostris in the low dose treatment was similar to that in the control. Although it was confirmed in the present study that the copepod-Bosmina interaction had been disturbed by carbaryl at the sublethal concentration, there was no detectable decline in B. longirostris density due to this disturbance.

Some lake ecosystems have many real problems due to anthropogenic impacts. Chemical pollution is one contributing factor to these problems, pesticides having great impact on the survival of the organisms in the system. Accurate evaluation of the impact of anthropogenic toxic chemicals on ecosystems is one of the most important goals for ecotoxicologists. It has been shown that the genera Bosmina and Bosminopsis are potentially more vulnerable to insecticides than Daphnia. Bosminid species commonly dominate the zooplankton communities in the eutrophic lakes, while Daphnia typically dominate oligotrophic lakes. It can therefore be considered that typical eutrophic plankton communities are more vulnerable to contamination than predicted by the results of the previous studies. 


\section{ACKNOWLEDGMENTS}

The authors thank staff of National Institute for Environmental Studies for their kind assistance during the experiment. We are grateful to Dr. H. Takagi and Dr. H. Mano for their helpful comments on this study. This study was supported by Grants-in-Aid to M. Sakamoto (No. 23510031) and to S. Kashiwada (No. 23310026) from Japan Society for the Promotion of Science.

\section{LITERATURE CITED}

Bossuyt BTA, Janssen CR. 2005. Copper toxicity to different field collected cladoceran species: intra- and inter-species sensitivity. Environ Pollut 136: 145-154.

Chang KH, Sakamoto M, Hanazato T. 2005. Impact of pesticide application on zooplankton communities with different densities of invertebrate predators: an experimental analysis using small-scale mesocosms. Aquat Toxicol 72: 373-382.

Finlay K, Beisner BE, Patoine A, Pinel-Alloul B. 2007. Regional ecosystem variability drives the relative importance of bottom-up and top-down factors for zooplankton size spectra. Can J Fish Aquat Sci 64: 516-529.

Gilbert JJ. 1988. Suppression of rotifer populations by Daphnia: a review of the evidence, the mechanisms, and the effects on zooplankton community structure. Limnol Oceanogr 33: 1286-1303.

Hanazato T. 1991a. Effects of repeated application of carbaryl on zooplankton communities in experimental ponds with or without the predator Chaoborus. Environ Pollut 74: 309-324.

Hanazato T. 1991b. Pesticides as chemical agents inducing helmet formation in Daphnia ambigua. Freshwater Biol 26: 419-424.

Hanazato T. 1995. Combined effect of the insecticide carbaryl and the Chaoborus kairomone on helmet development in Daphnia ambigua. Hydrobiologia 310: 95-100.

Hanazato T. 1998. Response of a zooplankton community to insecticide application in experimental ponds: a review and the implications of the effects of chemicals on the structure and functioning of freshwater communities. Environ Pollut 101: 361-373.

Hanazato T. 2001. Pesticide effects on freshwater zooplankton: an ecological perspective. Environ Pollut 112: 1-10.

Hanazato T, Dodson SI. 1993. Morphological responses of four species of cyclomorphic Daphnia to a short-term exposure to the insecticide carbaryl. J Plankton Res 15: 1087-1095.
Hanazato T, Kasai F. 1995. Effects of the Organophosphorus insecticide fenthion on phyto- and zooplankton communities in experimental ponds. Environ Pollut 88: 293298.

Hanazato T, Yasuno M. 1990. Influence of time of application of an insecticide on recovery patterns of a zooplankton community in experimental ponds. Arch Environ Contam Toxicol 19: 77-83.

Haney JF, Hall DJ. 1973. Sugar-coated Daphnia: a preservation technique for Cladocera. Limnol Oceanogr 18: 331333.

Havens KE, Hanazato T. 1993. Zooplankton community responses to chemical stressors: a comparison of results from acidification and pesticide contamination research. Environ Pollut 82: 277-288.

Havens KE. 1994. An experimental comparison of the effects of two chemical stressors on a freshwater zooplankton assemblage. Environ Pollut 84: 245-251.

Hense BA, Welzl G, Severin GF, Schramm KW. 2005. Nonylphenol induced changes in trophic web structure of plankton analysed by multivariate statistical approaches. Aquat Toxicol 73: 190-209.

Lürling M, Scheffer M. 2007. Info-disruption: pollution and the transfer of chemical information between organisms. Trends Ecol Evol 22: 374-379.

MacIsaac HJ, Gilbert JJ. 1989. Competition between rotifers and cladocerans of different body size. Oecologia 81: 295-301.

Mano H, Sakamoto M, Tanaka Y. 2010. A comparative study of insecticide toxicity among seven cladoceran species. Ecotoxicology 19: 1620-1625.

Moore M, Folt C. 1993. Zooplankton body size and community structure: effects of thermal and toxicant stress. Trends Ecol Evol 8: 178-183.

OECD. 2004. Test No. 202: Daphnia sp., acute immobilization test. In: OECD Guidelines for the Testing of Chemicals, Section 2: Effects on Biotic Systems.OECD Publishing, DOI: 10.1787/9789264069947-en.

Passino DRM, Novak AJ. 1984. Toxicity of arsenate and DDT to the cladoceran Bosmina longirostris. Bull Environ Contam Tox 33: 325-329.

Pereira JL, Gonçalves F. 2007. Effects of food availability on the acute and chronic toxicity of the insecticide methomyl to Daphnia spp. Sci Total Environ 386: 9-20.

Pérez-Ruiz T, Martinez Lozano C, Tomas V, Martin J. 2003. Flow injection chemiluminescence determination of carbaryl using photolytic decomposition and photogenerated tris (2,2'-bipyridyl)ruthenium(III). Anal Chim Acta 476: 141-148.

Preston BL. 2002. Indirect effects in aquatic ecotoxicology: 
implications for ecological risk assessment. Environ Manage 29: 311-323.

Preston BL, Cecchine G, Snell TW. 1999. Effects of pentachlorophenol on predator avoidance behavior of the rotifer Brachionus calyciflorus. Aquat Toxicol 44: 201-212.

R Core Development Team. (2011) R: A language and environment for statistical computing. R Foundation for Statistical Computing, Vienna, Austria. ISBN 3-90005107-0, URL http://www.R-project.org/. Accessed 1 May 2013.

Relyea R, Hoverman J. 2006. Assessing the ecology in ecotoxicology: a review and synthesis in freshwater systems. Ecol Lett 9: 1157-1171.

Sakamoto M, Chang KH, Hanazato T. 2005. Differential sensitivity of a predacious cladoceran (Leptodora) and its prey (the cladoceran Bosmina) to the insecticide carbaryl: results of acute toxicity tests. Bull Environ Contam Toxicol 75: 28-33.

Sakamoto M, Chang KH, Hanazato T. 2006. Inhibition of development of anti-predator morphology in the small cladoceran Bosmina by an insecticide: impact of an anthropogenic chemical on prey-predator interactions. Freshwater Biol 51: 1974-1983.

Sakamoto M, Chang KH, Hanazato T. 2007. Plastic phenotypes of antennule shape in Bosmina longirostris controlled by the physical stimuli from predators. Limnol Oceanogr 52: 2072-2078.

Sakamoto M, Hanazato T, Tanaka Y. 2009. Impact of an insecticide on persistence of inherent antipredator mor- phology of a small cladoceran, Bosmina. Arch Environ Contam Toxcol 57: 68-76.

Sarma SSS, Nandini S. 2006. Review of recent ecotoxicological studies on cladocerans. J Environ Sci Heal B 41: 14171430.

Stephen D, Balayla DM, Bécares E, Collings SE, FernándezAláez C, Fernández-Aláez M, Ferriol C, García P, Gomá J, Gyllström M, Hansson LA, Hietala J, Kairesalo T, Miracle MR, Romo S, Rueda J, Ståhl-Delbanco A, Svensson M, Vakkilainen K, Valentín M, Van de Bund WJ, Van Donk E, Vicente E, Villena MJ, Moss B, 2004. Continental-scale patterns of nutrient and fish effects on shallow lakes: introduction to a pan-European mesocosm experiment. Freshwater Biol 49: 1517-1524.

Tollrian R, Dodson SI. 1999. Inducible defenses in Cladocera: Constrains, costs, and multipredator environments. In The ecology and evolution of inducible defenses (Tollrian R, Harvell CD, eds). Princeton University Press, New Jersey, pp 177-202.

Van den Brink PJ, Ter Braak CJF. 1999. Principal response curves: analysis of time-dependent multivariate responses of biological community to stress. Environ Toxicol Chem 18: 138-148.

Van Wijngaarden RPA, Brock TCM, Van den Brink PJ. 2005. Threshold levels for effects of insecticides in freshwater ecosystems: a review. Ecotoxicology 14: 355-380.

Vesela S, Vijverberg J. 2007. Effect of body size on toxicity of zinc in neonates of four differently sized Daphnia species. Aquat Ecol 41: 67-73. 